\title{
Systematic characterization of germline variants from the DiscovEHR study endometrial carcinoma population
}

\author{
Jason E. Miller ${ }^{1}$, Raghu P. Metpally ${ }^{2}$, Thomas N. Person², Sarathbabu Krishnamurthy ${ }^{3}$, Venkata Ramesh Dasari ${ }^{3}$, \\ Manu Shivakumar ${ }^{2}$, Daniel R. Lavage ${ }^{2}$, Adam M. Cook ${ }^{3}$, David J. Carey ${ }^{3}$, Marylyn D. Ritchie', Dokyoon Kim²,4,5, \\ Radhika Gogoi ${ }^{3^{*}}$ and on behalf of the DiscovEHR collaboration
}

\begin{abstract}
Background: Endometrial cancer (EMCA) is the fifth most common cancer among women in the world. Identification of potentially pathogenic germline variants from individuals with EMCA will help characterize genetic features that underlie the disease and potentially predispose individuals to its pathogenesis.

Methods: The Geisinger Health System's (GHS) DiscovEHR cohort includes exome sequencing on over 50,000 consenting patients, 297 of whom have evidence of an EMCA diagnosis in their electronic health record. Here, rare variants were annotated as potentially pathogenic.

Results: Eight genes were identified as having increased burden in the EMCA cohort relative to the non-cancer control cohort. None of the eight genes had an increased burden in the other hormone related cancer cohort from GHS, suggesting they can help characterize the underlying genetic variation that gives rise to EMCA. Comparing GHS to the cancer genome atlas (TCGA) EMCA germline data illustrated 34 genes with potentially pathogenic variation and eight unique potentially pathogenic variants that were present in both studies. Thus, similar germline variation among genes can be observed in unique EMCA cohorts and could help prioritize genes to investigate for future work.
\end{abstract}

Conclusion: In summary, this systematic characterization of potentially pathogenic germline variants describes the genetic underpinnings of EMCA through the use of data from a single hospital system.

Keywords: Endometrial Cancer, Germline variants, Whole exome sequencing, DiscovEHR, TCGA, Uterine Cancer

\section{Background}

Endometrial cancer (EMCA) is the most common cancer of the female reproductive tract with an estimated 62,230 new cases and 11,350 deaths estimated in 2018 in the United States alone [1]. The treatment of EMCA has become a major issue for the health-care system because of its increasing incidence and death rate over the past two decades [2]. Traditional U.S. categorization of EMCA is based into two broad classifications from the National Comprehensive Cancer Network (NCCN), type 1 and 2, based on histology, steroid hormone receptor expression, and prognosis [3]. Type 1 is more common, and is characterized by endometrioid histology, is

\footnotetext{
* Correspondence: radhika.gogoi@gmail.com; rpgogoi@geisinger.edu

${ }^{3}$ Weis Center for Research, Geisinger Medical Center, Danville, PA 17822, USA Full list of author information is available at the end of the article
}

estrogen potentiated, estrogen receptor (ER) and progesterone receptor (PR) positive, and generally carries a favorable prognosis [2-4]. Type 2 is ER/PR negative and carries a much poorer prognosis [4]. Epidemiological studies confirm an association of EMCA with obesity [5], early menarche, late menopause, nulliparity, exogenous factors (estrogen only use), and other lifestyle factors related to low physical activity [3, 6]. While type 1 is treated by surgery followed by radiation for high risk features, type 2 is treated by radiotherapy or surgery followed by systemic chemotherapy. Nevertheless, treatment has high variability in efficacy and side effects [7]. Of those diagnosed with endometrial cancer, $90 \%$ is sporadic while the remaining $10 \%$ is hereditary [8]. Lynch syndrome or hereditary nonpolyposis colorectal cancer (HNPCC), is an autosomal dominant disorder 
that not only represents an increased risk of colon cancer, but an increased risk also of EMCA for women [8]. Additionally, it is characterized by a mutation in one of a group of DNA mismatch repair genes (MSH1, MSH2, MLH6, PMS2 or EpCAM) [9, 10]. However, not all families that meet clinical criteria for Lynch syndrome have an identifiable mutation in these genes [11].

DNA sequencing can identify genetic variants associated with different types of cancer. For most cancers, EMCA included, somatic mutations and matched controls have been used to generate insights as to the potential pathogenicity of the variants (Single Nucleotide Variants (SNVs) and insertions/deletions (indels)) [1215]. A motivating factor behind studying the germline is supported by the "two hit hypothesis" [16], which describes when a tumor suppressor gene is inactivated initially by a germline mutation followed by a somatic mutation on another allele that leads to tumorigenesis. While there remain open questions related to this hypothesis, the investigation of loss of heterozygosity (sometimes referred to as "allelic loss") of a tumor suppressors has provided support for this theory [17-20]. Furthermore, the analysis of germline variants has improved the detection of driver mutations when somatic variants have also been available [21, 22]. The Cancer Genome Atlas (TCGA) illustrated that cancer susceptibility genes could be identified across several cancer types using data produced from the germline, including but not limited to EMCA, by searching for enrichment of rare variants that resulted in truncations [23]. Moreover, germline data has been used to identify genes previously unknown to be associated with ovarian cancer [24]. Thus, investigating germline variants serves as a tool to assist in the characterization of potential genetic drivers underlying cancer.

Though a number of studies have generated significant findings related to the underlying genetic architecture of EMCA using only germline or matched samples [22, 25, 26], there are growing number of hospital-systems and country-wide genetic studies that have primarily generated germline level data [25]. These projects offer new ways to potentially investigate EMCA. For instance, our group and others have reported the use of patient-participant billing codes extracted from electronic health records (EHR) and common variants from patient-participants to perform association studies [26-28]. A recent study identified predisposition mutations in an EMCA cohort using a multiplex PCR panel [29]. However, it is largely unknown if comparing rare germline variants from whole exome sequencing between case and control cohorts from a single institution can reproduce what is known and identify novel genetic underpinnings related to EMCA, however it is unmistakable that this strategy could be used by a variety of institutions and in other disease contexts.
In 2007, Geisinger Health System (GHS) launched MyCode, a system-wide biobanking program to link samples and electronic health record (EHR) data for broad research use [30]. GHS is an integrated health system, serving a stable patient population, and with longitudinal EHR data that documents patients' treatment and clinical outcome [30]. These features of MyCode can be used to compare genetic variants in individuals with and without cancer in a large clinical population. Recently, we reported the results of analysis of more than 50,000 MyCode DNA samples that had undergone whole exome sequencing (WES) as part of the DiscovEHR study [31]. Among DiscovEHR participants were 297 patient-participants who had been diagnosed with EMCA. We hypothesized that characterization of germline variants in WES data in a cohort of participants with EMCA would lead to insights into the genetic basis of EMCA. In this study, we describe the identification of rare, potentially pathogenic variants in DiscovEHR EMCA, a non-cancer cohort, and other hormone related malignancy cancer cohort from a single hospital system.

\section{Methods \\ Patient-participant cohorts}

This study consisted of GHS patients who consented to participate in the MyCode Community Health Initiative [30]. MyCode participants agree to provide samples for broad research use and linking of samples to data in the EHR database as part of the DiscovEHR study. EMCA participants were identified through ICD-9 code and then validated through manual chart review of the pathology report $(N=297)$. Demographic information including age and BMI at the time of diagnosis, histology, stage, treatment and overall survival were obtained from the EHR by a Gynecologic oncologist. The elderly non-cancer control cohort (NCC, $N=2120$ ) consisted of females older than 70 years old, with no history of cancer diagnosis (absence of ICD9/ICD10 encounter/problem list diagnosis codes related to cancer). The other hormone related malignancy $(\mathrm{OHRM})$ cohort $(N=1463)$ was generated by identifying female participants in DiscovEHR who have ICD-O (international classification of diseases for oncology) codes related to breast (c50x) or ovarian cancer (C48.0, C48.1, C48.2, and C56.9). For further analysis, stages 1 and 2 were defined as "early", while 3 and 4. EMCA were defined as "late". The FIGO and TNM staging systems were used. U.S. treatment guidelines were from the National Comprehensive Cancer Network (NCCN) [3].

\section{Exome sequencing and variant calling}

WES data was collected from MyCode participants from the DiscovEHR database and processed with slight modifications [31]. Raw reads were aligned using BWA-mem. 
Mapping and alignment to GRCh37.p13 was then performed and variants were called using Genome Analysis Toolkit (GATK) "best practices" [32]. Described in brief, after indel realignment, and base recalibration using BQSR (Base Quality Score Recalibration) for the entire GHS cohort, and gVCFs (genomic VCF) are called. The gVCF files were then combined into a merged gVCF and the recalibrated haplotypes are then called using GATK HaplotypeCaller. These gVCFs were then filtered for high quality variants using variant quality score recalibration (VQSR). Another filtering step was used to obtain variants with genotype quality $\geq 20$ [33].

\section{Variant filtration and annotation}

Participant VCFs were selected from the DiscovEHR study project that were associated with each cohort. Additional file 1: Figure S1 summarizes the process by which variants were characterized as potentially pathogenic. Variants that fell within the genomic boundaries (RefSeq annotations using VEPv91) of genes (635 unique genes) from the TARGET database (https://software. broadinstitute.org/cancer/cga/target (version 3)) and/or Cancer Gene Census (CGC, https://cancer.sanger.ac.uk/ census (downloaded on March 7th, 2017)) were carried forward [34]. Variants were then filtered based on their likelihood of being pathogenic [35-37]. Those variants with 1 or more star or called as either "likely pathogenic" or "pathogenic" in Clinvar [35] or were "High" in VEP (Variant Effect Predictor) were also included [36]. Variants with a minor allele frequency greater than $1 \%$ in either the DiscovEHR cohort, Exome Aggregation Consortium (EXaC [38]), NHLBI GO Exome Sequencing Project (ESP [39]) and 1000 Genomes Project were removed [40]. This procedure was based on recommendations from the American College of Medical Genetics and Genomics [41]. Variants that made it through this pipeline were then included in downstream analysis.

To reformat, summarize, and visualize the data, the following $\mathrm{R}$ packages were used: ggplot2, dplyr, tidyr, GenVisR, and reshape2. Typically, only one transcript isoform made it through the variant filtration and annotation pipeline. Variants were grouped into three categories, synonymous, non-synonymous, and predicted loss of function (pLoF) for further analyses. These definitions adhere closely to previous work [31]. If the VEP consequence was synonymous_variant the variant was categorized as "synonymous". If the VEP consequence was missense_variant, stop_retained_variant, initiator_codon_variant, inframe_deletion, inframe_insertion, or splice_region_variant the variant was categorized as "non-synonymous". If the VEP consequence was stop_gained, stop_lost, start_lost, splice_donor_variant, splice_acceptor_variant, frameshift_variant, disruptive_inframe_deletion, disruptive_inframe_insertion, or protein_protein_contact the variant was categorized as "pLoF". If the splice_region_variant co-occurred with a synonymous, missense, or pLoF variant it was called as synonymous, missense, or pLoF, respectively. If the variant was located in lower confidence region (e.g. UTR, upstream_gene_variant, downstream_gene_variant, intron_variant, or TF) the variant was excluded from analysis. Variants were excluded if they were not targeted by probes in the exome-capture process. The average EMCA patients with a variant per control group patient was calculated for each gene by: (Variants ${ }_{\mathrm{GeneX}}$ in EMCA patients $/ 297$ EMCA patients)/ (Variants ${ }_{\text {GeneX in control }}$ patients $/ 2120$ control patients). The average OHRM patients with a variant per control group patient was calculated for each gene by: (Variants GeneX in OHRM patients $/ 486$ OHRM patients)/ (Variants ${ }_{\mathrm{GeneX}}$ in control patients $/ 2120$ control patients). Graphical and statistical analysis was performed in R.

\section{Comparison to TCGA Germline Uterine Cancer data}

Uterine Corpus Endometrial Carcinoma (UCEC) germline and somatic variants were retrieved from the Broad GDAC Firehose (https://gdac.broadinstitute.org). The germline VCFs were processed in the same manner as the DiscovEHR data (see previous section) to identify variants in the list of genes from TARGET and CGC (Additional file 1: Figure S1).

\section{Results}

\section{Demographics and histology of cancer and non-cancer cohorts}

The Geisinger MyCode community health initiative includes over 150,000 participants who have agreed to provide blood samples for broad research use [30] (www.geisinger.edu). In the first 50,726 participants to undergo WES analysis, we identified 297 patients with a diagnosis of EMCA (Additional file 5: Table S1). The average age of individuals with EMCA in our study was 61 , which is similar to the national average, e.g. 60 years old (cancer.org). While most women diagnosed with EMCA in the U.S. are older than 45, the age range is from 27 to 87 in this study. However, $94 \%$ of our samples came from individuals older than 45 , suggesting our cohort represents a similar age as is seen nationally. The DiscovEHR cohort as a whole has an average BMI of 30 $\mathrm{kg} / \mathrm{m}^{2}$ [31], and the average BMI in the EMCA cohort was $38 \mathrm{~kg} / \mathrm{m}^{2}$ (Additional file 5: Table S1). This result is consistent with previous research which has found an increased risk of uterine-related cancer with increasing BMI [42].

Chart review revealed that most EMCA cases were of endometrioid histology, and early stage and grade (Additional file 5: Tables S2-S5). An elderly female non-cancer cohort (NCC, $N=2120$ ) was used to identify potential EMCA-associated genetic variants by selecting 
older individuals who have no record of cancer. Additionally, a separate hormone related malignancy (OHRM) cancer cohort $(N=1463)$, consisting of individuals with breast or ovarian cancer, was also analyzed. Since the OHRM cohort represents estrogen-driven cancer etiology similar to EMCA, it provides an opportunity to identify EMCA-specific variants as opposed to other hormone-related cancers. Our control cohort has an average BMI of $27 \mathrm{~kg} / \mathrm{m}^{2}$ which while overweight, is on average lower than the entire DiscovEHR cohort as a whole. The average BMI of the OHRM cohort is 29 $\mathrm{kg} / \mathrm{m}^{2}$, which is close to an obese classification, and consistent with the association between obesity and cancer [43]. The total number of patients with endometrioid EMCA was 265, while 2 had non-endometrioid EMCA that are grade 3 (Additional file 5: Tables S2 and S4). The DiscovEHR and TCGA cohorts had similar distributions of grade 2 samples, but TCGA had many more grade 3 , and fewer grade 1 (Additional file 5: Table S4). Grade one and two U.S. estimates were similar to the other cohorts [44]. Compared to U.S. estimates, TCGA has more grade 3 as a percentage and DiscovEHR had a smaller percentage (Additional file 5: Table S4). Downstream analysis of all grades and stages are performed together unless otherwise noted. The average time of follow up after diagnosis was 6 years, and most patients were disease free in the absence of further therapy (Additional file 5: Tables S1 and S5). All patients who were stage 3 or 4 received surgery (Additional file 5: Table S5).

\section{Identifying rare pathogenic germline variants in cancer and non-cancer cohorts}

To identify variants that are relevant to EMCA a bioinformatics pipeline was created to identify variants that are predicted to be likely pathogenic or pathogenic (Additional file 1: Figure S1). Only genes that were in the TARGET or Cancer Genome Census (CGC) database (635 genes total) were included to increase confidence that variants we identify are related to cancer or therapeutic outcomes $[34,45]$. Variants were binned into the 635 TARGET and/or CGC genes, then annotated using ClinVar and variant effect predictor (VEP) to identify likely pathogenic or pathogenic variants. Only variants with minor allele frequency (MAF) less than $1 \%$ in the Geisinger DiscovEHR population, and not greater than $1 \%$ in any total or sub population from Exome Aggregation Consortium (ExAC [38]), Exome Sequencing Project (ESP [39]), or 1000 genomes project [40] were included. This process identified variants in 28 to $32 \%$ of the participants across the three cohorts (Table 1). The number of total and unique variants across the three cohorts is summarized in Table 1. Therefore, all variants described from here forward are considered likely pathogenic or pathogenic.

\section{Exploring EMCA histology and rare variants}

The variants were visualized in a participant centric graph using a co-mutation plot, sometimes referred to as a waterfall plot (Fig. 1). There did not appear to be enrichment of a specific EMCA histology, and/or patient status among genes that are often mutated (i.e. $A P O B E C 3 B$ ) compared to those with few variants (i.e. ARID1B) (Fig. 1). Generally, most participants had variants associated with the most common diagnosis (i.e. endometrioid histology). Of the 297 participants with EMCA, six had been previously clinically diagnosed with Lynch syndrome, however, only two contained a rare pathogenic variant that met our workflow criteria (Additional file 2: Figure S2). The participants had a frameshift variant in $M S H 2$, a Lynch syndrome gene [46].

\section{Characterization of pathogenic germline variants between EMCA, OHRM and NCC cohorts}

The variants across the three cohorts included a mixture of indels and single nucleotide variants (SNVs) (Fig. 2a). Based on criteria (see methods) from previous work, SNVs and indels were grouped together based on whether they were synonymous, non-synonymous or variants that are predicted to cause a loss of function (pLoF). No synonymous variants were identified as potentially pathogenic. Most of the non-synonymous variants were missense, while most pLoF variants were stop gained or frameshift (Fig. 2b). Non-synonymous and pLoF made up similar percentages of the variants in all three cohorts (Fig. 2b). In a previous analysis of variants from more than 50,000 DiscovEHR participants, non-synonymous and synonymous variants made up a greater number of sites with variants than did pLoF [31]. However, after our rare variant annotation algorithm (Additional file 1: Figure S1) was applied to the cohorts in this study, synonymous variants were filtered out leaving mostly pLoF and some non-synonymous variants (Fig. 2a). These results demonstrate that the algorithm enriches for rare variants with potential pathogenic influence.

The number of unique variants and genes that contained variants are described in Table 1 . The genes overlapping between the 3 cohorts is shown in Fig. 2c. While there was considerable overlap between the cohorts, 4 genes were unique to the EMCA cohort (e.g. CDK4, LIFR, MALT1, and MSH2). Two genes were identified only in the EMCA and OHRM cohorts (e.g. PMS2 and TMPRSS2). A comparison of unique variant loci showed differences among the cohorts compared to gene-wise comparison (Fig. 2d). The vast majority of genes present 
Table 1 Summary of rare pathogenic variant distribution across cohorts

\begin{tabular}{llll}
\hline & EMCA & NCC & OHRM \\
\hline Participants $^{\mathrm{a}}$ & 297 & 2120 & 1486 \\
Participants with variants after filter $^{\mathrm{b}}$ & $85(28.6 \%)$ & $628(29.6 \%)$ & $462(31.1 \%)$ \\
Genes with variants after filter $^{\mathrm{c}}$ & 62 & 211 & 205 \\
Loci with variants $^{\text {d }}$ & 73 & 485 & 371 \\
Rare variant burden across participants in each cohort $^{\mathrm{e}}$ & 99 & 791 & 593 \\
\hline
\end{tabular}

Summary level data of participants from WES and rare variant analysis. The total number of participants included in each cohort $\left({ }^{\mathrm{a}}\right)$. The total number of participants from " $a$ " that had at least one rare variant that met criteria from Fig. 1 workflow, $\left({ }^{b}\right)$. The number of genes with at least one rare variant that met workflow criteria $\left({ }^{\mathrm{c}}\right)$. The number of unique variants present after filtering using the bioinformatics pipeline in Fig. $1\left({ }^{\mathrm{d}}\right)$. The total number of unique and nonunique rare variants present across the participants in the cohort ( ${ }^{\mathrm{e}}$ )

in the EMCA cohort could be found in the other two cohorts. This result suggests that to identify more genes with EMCA relevant genetic variation, an alternate metric such as calculating the difference in frequency between the genes with rare pathogenic variants in EMCA and the other cohorts could be useful.

\section{Comparing rare variant burden between cancer and NCC cohorts}

We next evaluated whether analysis of variant burden could identify genes that are more likely to be associated with EMCA. The number of variants per individual was compared in the EMCA and NCC cohorts (Table 1) (see

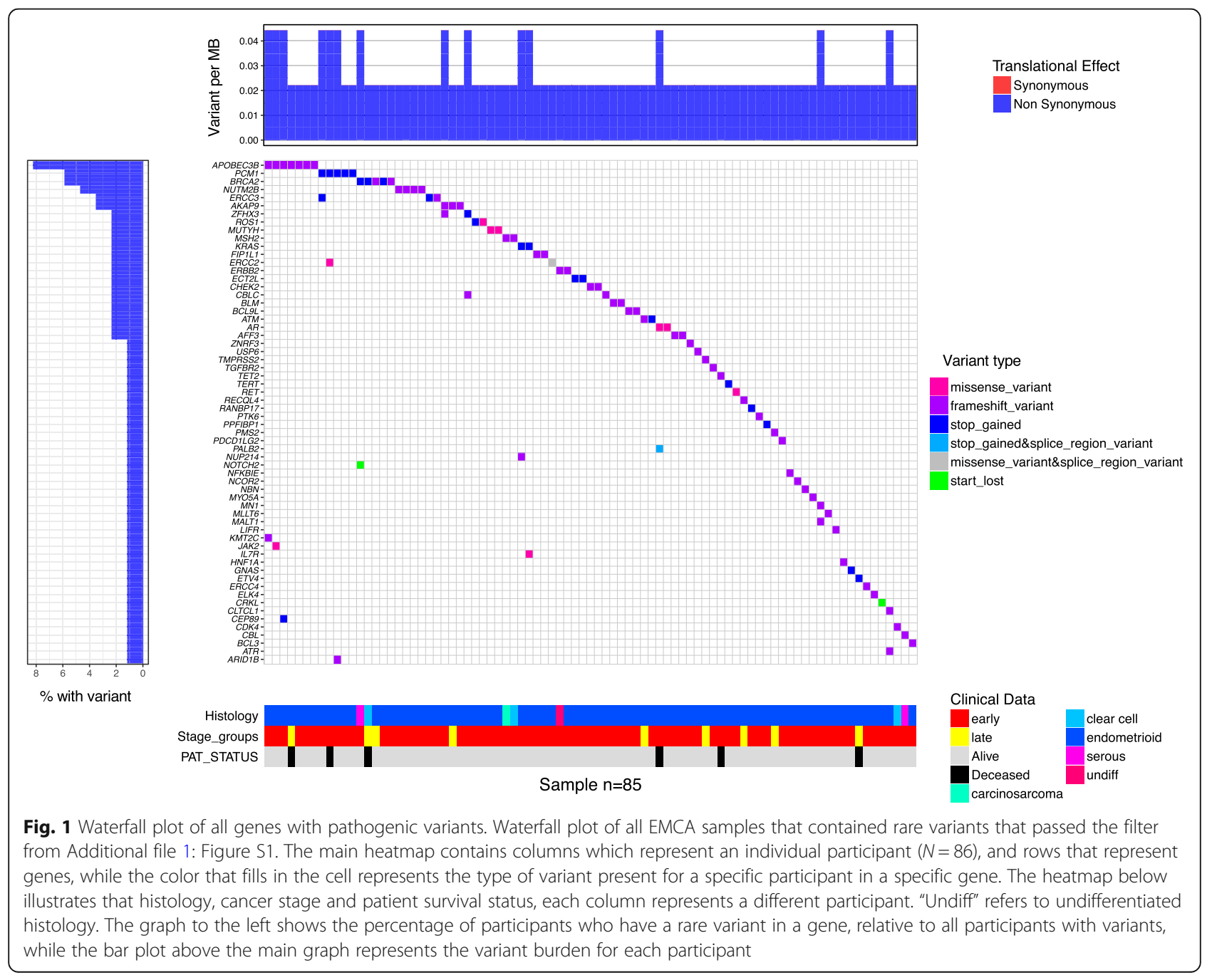



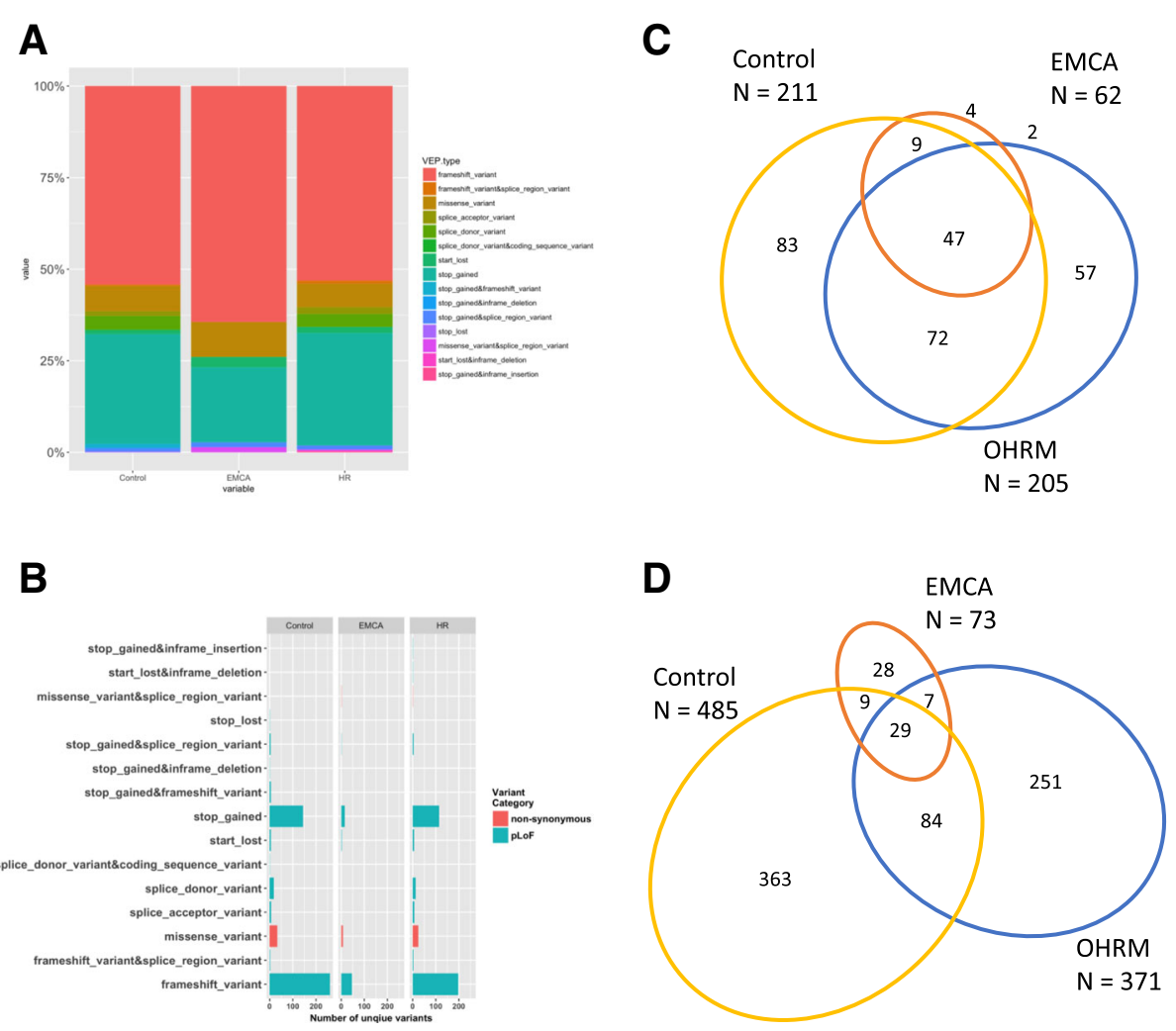

Fig. 2 Distribution of types of rare variants across cohorts. The total number of unique variants are represented by their VEP annotation. Additionally, each type of variant is grouped by the variant category (a). The percentage of each variant category represented in each cohort (b). The overlap between genes with variants represented in each cohort (c). The number of unique loci (variants) that overlap between each cohort (d)

methods). Only genes with at least two variants across the EMCA and NCC cohorts were considered. There was only one gene, $E R C C 2$, with a higher burden of non-synonymous variants in EMCA participants compared to the NCC cohort (Fig. 3a and b). Seven genes showed at least a 2-fold enrichment in the EMCA cohort compared to NCC using pLoF variants (Fig. $3 \mathrm{c}$ and d; ECT2L, BLM, $A P B E C 3 B, P C M 1, Z F H X 3, E R C C 3$, and AFF3). To verify that the increased burden among EMCA participants is specific to EMCA and not hormone related cancers, the burden between OHRM and the NCC cohort was also measured (Additional file 3: Figure S3 and Additional file 4: Figure S4). Only RNF213 was found to have increased pLoF variant burden in the OHRM cohort, however this was not observed in the EMCA cohort (Additional file 4: Figure S4 and 3D). Since there were no shared genes with at least 2-fold enrichment among EMCA and OHRM relative to the NCC, all of the genes with high variant burden in the EMCA cohort are unique to EMCA compared to the OHRM cohort. We used a difference of proportions test to evaluate the differences between EMCA and NCC or OHRM and NCC (Additional file 6). While several genes had a $p$-value less than 0.05 , they were not significant after correcting for multiple tests. These results suggest a larger sample size or different experimental design is necessary for capturing statistically significant results (Additional file 6).

\section{DiscovEHR germline variants are reproduced in TCGA study}

We also compared germline variants in the DiscovEHR EMCA cohort to the uterine cancer samples in the Cancer Genome Atlas (TCGA) Research Network. TCGA performed a comprehensive, multiplatform analysis of type 1 and 2 EMCA using array- and sequencing-based technologies, including WES [47]. DiscovEHR and TCGA had 89 and $81 \%$ endometrioid histology, respectively, however median follow-up time was much greater for the DiscovEHR EMCA cohort (67 months) relative to TCGA (32 months) [47]. A total of 553 rare variants from TCGA germline variants met the criteria of the workflow in Fig. 1 that was applied to the EMCA DiscovEHR data (Fig. 4). Of these likely pathogenic variants, only eight were also found in the DiscovEHR EMCA cohort (Fig. 4a). The 73 Geisinger Health System germline variants were in 62 different genes, 34 of which were also identified in the TCGA EMCA cohort (Fig. 4b). Of the eight genes that had higher burden 

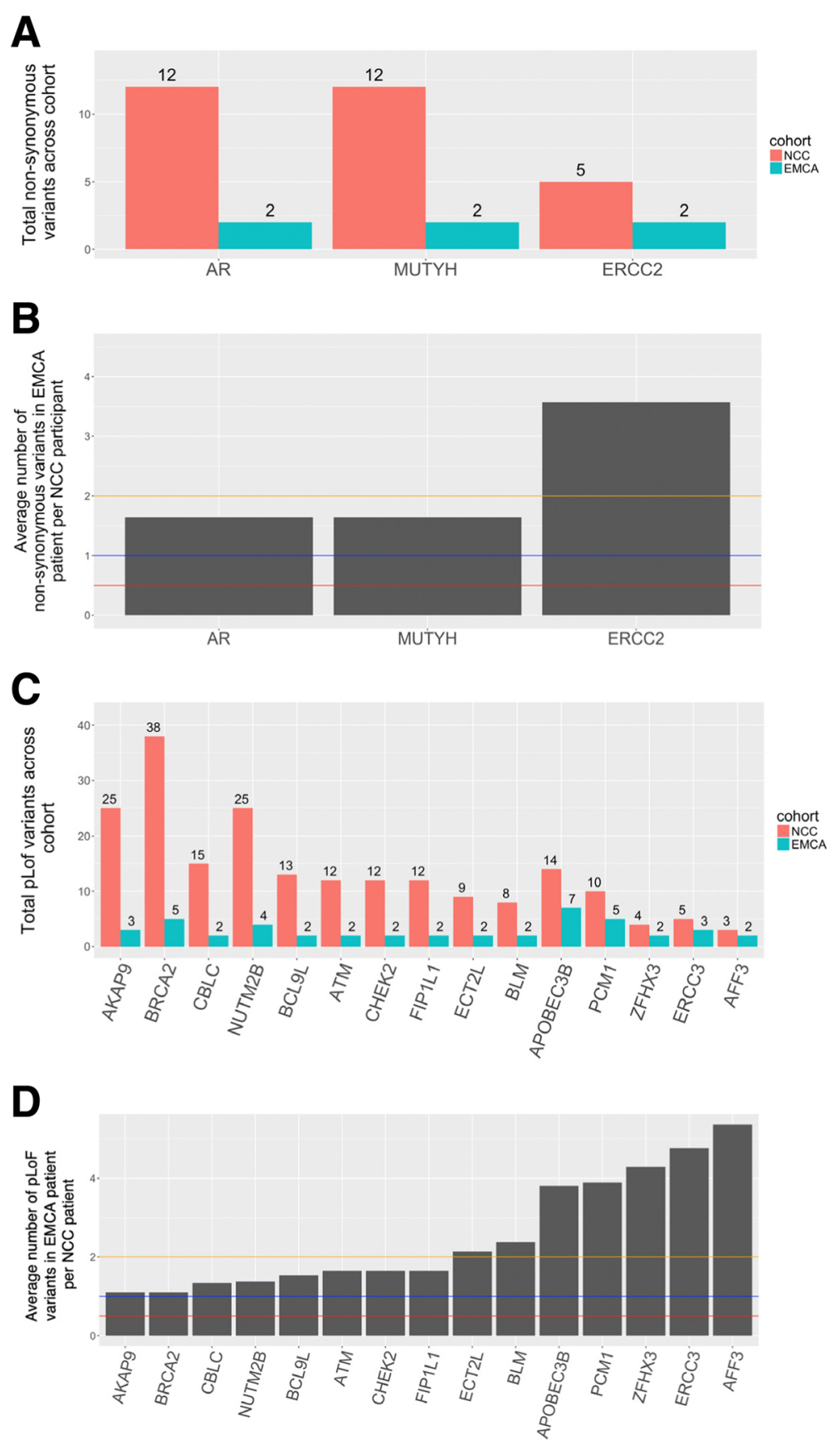

Fig. 3 Non-synonymous and pLoF variants among EMCA to non-cancer control cohort. a For each gene with at least two variants in both EMCA and NCC, the ratio of non-synonymous variants across the EMCA cohort was divided by those in the NCC after adjusting for differences in cohort size. Orange, blue and red lines are used to delineate 2, 1 and 0.5 fold EMCA burden relative to the NCC cohort. b The total number of rare non-synonymous variants from each cohort for each gene. $\mathbf{c}$ and $\mathbf{d}$ the same as $\mathbf{a}$ and $\mathbf{b}$, respectively, except pLoF variants were used

in the EMCA cohort relative to NCC, four (e.g. $B L M$, $E C T 2 L, E R C C 2$, and ERCC3) were present in the 34 genes represented in both DiscovEHR and TCGA. Additionally, MALT1 and $M S H 2$, two genes that were only observed to have variants in the EMCA only cohort (Fig. 2c) were among the 34 genes that replicated. These results support the concept that rare variants binned into genes can do a better job of capturing replication relative to being located in the same loci across individuals [48]. Furthermore, the relatively high congruency between the two studies at the gene level highlights how germline variation in EMCA participants is reproducible for this study. Only one variant (e.g. nonsense variant in the gene ETV4) was found to replicate between the 


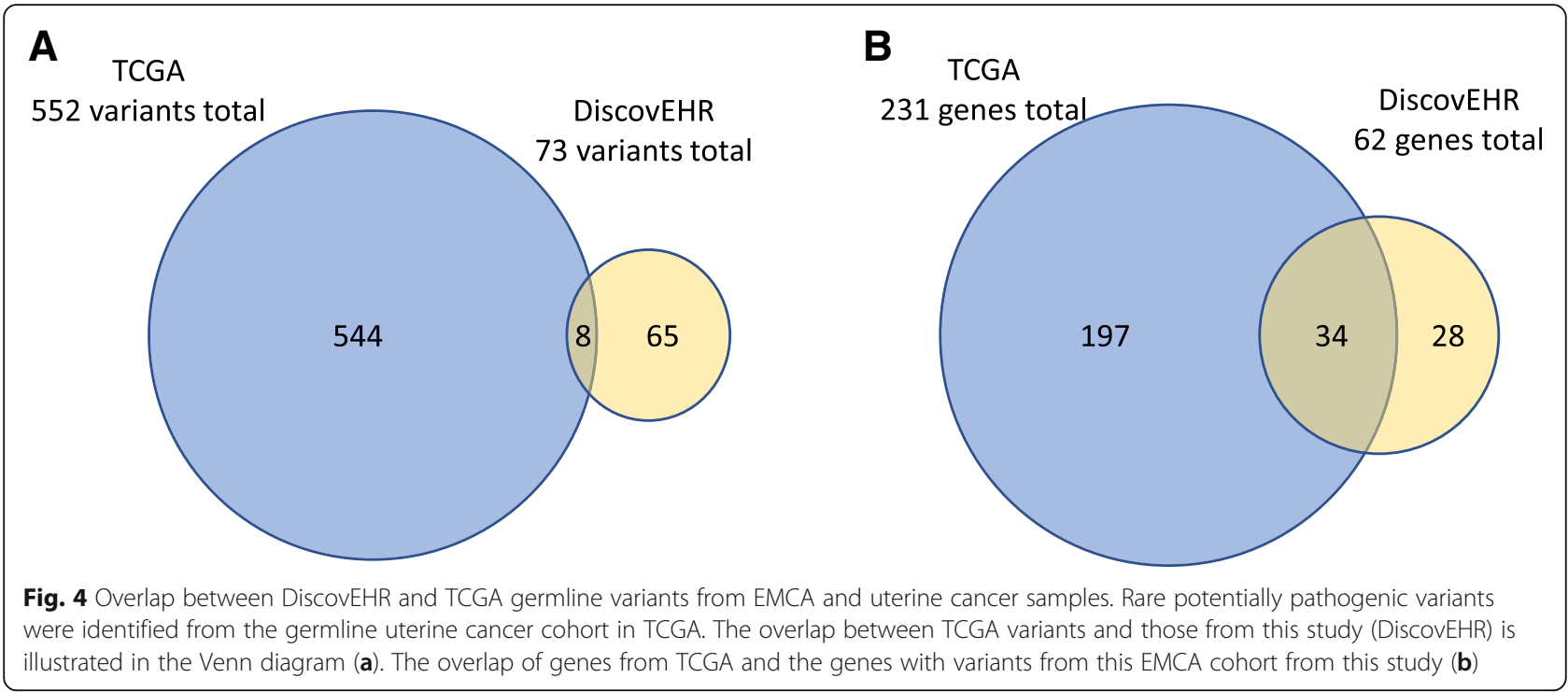

Geisinger germline results and the TCGA somatic mutations.

\section{Discussion}

While significant progress has been made identifying somatic variants associated with EMCA [47, 49], a number of studies have pointed to the usefulness of looking at germline variants as an alternative means to characterize the genetic etiology of EMCA [29, 50]. In this work, we describe the landscape of germline variants from participants diagnosed with EMCA using the WES data from the DiscovEHR study. We identified rare variants in 4 genes that were unique to the EMCA cohort. Rare variants in the genes CDK4, LIFR, MALT1, and $M S H 2$ were identified in the DiscovEHR EMCA cohort but not NCC and OHRM. CDK4 promotes progression of the cell cycle and increased expression is observed in 34-77\% of endometrioid endometrial carcinoma (EEC) [51]. Additionally, the specific activity of CDK4/6 has been illustrated to be a biomarker for predicting recurrence of EEC in pathologically low-risk group of patients [52]. Recent evidence suggests the expression of leukemia inhibitory factor receptor (LIFR) affects multiple signaling pathways in the endometrium of patients with adenomyosis during the window of implantation for in vitro fertilization [53, 54]. Chromosomal translocation of MALT1 associated with MALT lymphoma is often restricted to the endometrium [55]. Individuals with germline mutations in $\mathrm{MSH} 2$ can be diagnosed with Lynch Syndrome, which in turn is associated with a high risk of colorectal cancer, including but not limited to EMCA [56]. Since an extensive literature exists on all but MALT1, it suggests further work should be devoted to teasing out its connection to EMCA.
$A P O B E C 3 B$ was the gene most often identified as having a rare variant among EMCA participants. $A P O$ $B E C 3 B$ is a member of a gene family, which consists of seven members, that cause cytosine-to-uracil deamination of single-stranded DNA [57]. These cytosine deaminases mediate intrinsic immunity to retroviruses and endogenous retrotransposons $[58,59]$. Mutation or low expression of the tumor suppressor p53 is associated with an increase in expression and activity of APO$B E C 3 B$ in endometrial cancer and other types of cancer as well [57]. PCM1 and BRCA2 were found to have the second most rare variants among EMCA participants ( $N=5$ each). PCM1 codes for a protein that is responsible for anchoring microtubules to centrosomes and has previously been associated with thyroid cancer, leukemia, and T-cell lymphoma [60-63]. However, PCM1 does not have a previously known connection to EMCA, suggesting it is a novel candidate for investigating its relationship to EMCA. Alternatively, previous work found carriers of $B R C A$ mutations, especially $B R C A 1$, had increased risk of EMCA [64].

Our data also suggests that relying only on the EMCA cohort or looking for variation that does not overlap with other cohorts to investigate the genetic etiology of EMCA is problematic, as several genes can be missed. Whereas a burden-based approach appeared to be a superior method for finding genes that have EMCA-associated variation. Only four genes were identified as having variants unique to the EMCA cohort, whereas the burden-based approach identified eight genes, and importantly, controlled for genes which have many variants in the NCC or OHRM cohorts. Together, these results suggest a burden-based approach can lead to the identification of more genes that help characterize germline variation and account for genes that are more prone to germline variants in EMCA 
patient-participants. Several genes that were identified using the burden-based approach regulate DNA repair or transcription. For instance, $E R C C 2$ and $E R C C 3$ are a part of the general transcriptional machinery TFIIH and nucleotide excision repair [65]. The transcription factor $A F F 3$ associated with lymphoid development and neuronal differentiation [66, 67]. ZFHX3 is also a transcription factor, it acts as a tumor suppressor in multiple cancer types $[68,69]$. Though these observations are consistent with a previous body of work which has found a connection between developmental processes and cancer [70], we found that rare variants in these genes may be acutely important for understanding EMCA.

To evaluate how reproducible and biologically relevant the rare variants are among EMCA cohorts we compared the variation observed in our cohort to that were seen in TCGA germline samples. This comparison illustrates consistent variation at the gene level, but not at the variant level. Moreover, these results support a known feature of genetic variation, that while rare variants are infrequently observed at the same exact loci across individuals, trends do appear once the rare variants are analyzed in the context of a gene or pathway [48]. Replication is especially important to look for here since rare variants may be spurious [48]. Thus, our ability to find reproducible variation increases confidence that variation within genic regions will be helpful for characterizing EMCA on a molecular level. The genes identified may play a role in the development of EMCA, therefore this work may provide a useful strategy for identifying possible therapeutic targets either through drug or mutation. While the EMCA cohort from DiscovEHR had a similar distribution of stages, there were more grade 3 samples in TCGA. Thus, when comparing the two, the interpretation should consider that the overlap was between samples of varying stages and grades. As discussed below, larger sample sizes in the future may allow for stratified analyses. Nonetheless, because most EMCA diagnoses in the U.S. are estimated to be grade $1 / 2$ and stage 1 , it is possible that results from DiscovEHR can be generalized.

Previous work has demonstrated how individual germline variants contribute to EMCA [49]. Here we utilized WES data obtained from participants of a single health system as part of the DiscovEHR study. By performing a manually curated chart review, there was added confidence in the identification of those diagnosed with EMCA. An advantage of performing the study using the DiscovEHR cohort is that due to the location, the participants have similar demographics, suggesting that ancestry and socio-economic status likely play a smaller role in explaining variation between participants in the DiscovEHR cohort. Conversely, future studies with larger cohorts that have less homogenous ancestry and cancer types could provide important insights into the etiology of EMCA across different subpopulations. A larger cohort, with more individuals across ancestries, stages, treatments, and outcomes could also provide a useful platform for characterizing our genetic understanding of EMCA and performing more risk assessment related analyses.

An outstanding obstacle in the field of EMCA research is the difficulty in risk assessment for individuals who already have EMCA. While this work did not address that issue, we look forward to future work that applies the use of EHR and WES to that specific clinical application. For instance, obesity is a risk factor, but it could also independently be associated with the variant burden. Future studies will need to be carefully designed in order to test for this effect. Having said that, the genes identified in this study may play a role in the initiation or inception of EMCA, and with experimental functional validation could be prioritized as potential therapeutic targets. In summary, this study suggests that WES from a single hospital system can provide useful insights into the molecular signatures for which to distinguish variation in EMCA from that in NCC and OHRM cohorts. Moreover, these genes and variants may help identify causal links to the pathogenesis of EMCA.

\section{Conclusions}

The purpose of this study was to investigate the differences and similarities between potentially pathogenic germline variants among patient participants from EMCA, OHRM, and NCC cohorts using EHR. Although larger sample sizes or alternative approaches will be needed to capture statistically significant associations, a number of conclusions can be made from this analysis which characterize EMCA in new ways. Binning potentially pathogenic variants from the DiscovEHR WES data into genes illustrated greater overlap between cohorts compared to looking at the overlap of loci. Only four genes had variants unique to the EMCA cohort, where as a burden-based approach detected eight genes that were enriched with potentially pathogenic variants. High concordance between the DiscovEHR and TCGA cohorts illustrates that reproducible potentially pathogenic germline variation can be observed in multiple studies. In summary, there are many overlapping genetic features between EMCA and non-EMCA cohorts, however, a burden-based approach can best help to characterize the genetic underpinnings of EMCA.

\section{Web resources}

Variant sites and frequencies with basic annotations from the DiscovEHR study is hosted in the following database and webserver: www.discovehrshare.com. Further information concerning the reproduction of results described in this article is available upon reasonable request and subject to a data use agreement. The TARGET database (https://software.broadinstitute.org/cancer/cga/target 
(version 3)) and Cancer Gene Census (CGC, https://cancer.sanger.ac.uk/census (downloaded on March 7th, 2017)) were used in this work. Uterine Corpus Endometrial Carcinoma (UCEC) germline and somatic variants were retrieved from the Broad GDAC Firehose (https:// gdac.broadinstitute.org).

\section{Additional files}

Additional file 1: Figure S1. Bioinformatics workflow for detecting pathogenic variants. Variants are first binned into the 635 genes from TARGET and CGC. They are then carried forward if they meet certain criteria from multiple variant annotation databases (e.g. Clinvar and VEP). Finally, variants with a MAF $>1 \%$ In the MyCode cohort, or any total or subpopulation from EXaC, ESP and 1000 genomes. (PPTX $43 \mathrm{~kb}$ )

Additional file 2: Figure S2. Variants in Lynch Syndrome Participants. The main figure is a heatmap of columns for each of the 6 participants who have been previously diagnosed with Lynch syndrome. The rows represent the genes in which these variants reside in and the color is the type of variant (see key to right). The variant burden for each participant and the individual genes are represented has histograms above and to the left of the main figure, respectively. Below the main heatmap is another diagram which illustrates the histology, stage and patient status along with reporting that all 6 participants had a Lynch diagnosis. (PPTX $50 \mathrm{~kb}$ )

Additional file 3: Figure S3. Non-synonymous variants among OHRM and NCC cohorts. (A) For each gene with two variants in both cohorts, the ratio of non-synonymous variants across the EMCA cohort was divided by those in the NCC after adjusting for differences in cohort size. Orange, blue and red lines are used to delineate 2, 1 and 0.5 fold EMCA burden relative to the NCC cohort. The graph inset represents the raw number of variants at each gene between the OHRM and NCC cohort. (B) The number of rare non-synonymous variants from each cohort. (PPTX $73877 \mathrm{~kb}$ )

Additional file 4: Figure S4. pLoF variants among OHRM and NCC cohorts. For each gene with two variants in both cohorts, the ratio of non-synonymous variants across the OHRM cohort was divided by those in the NCC after adjusting for differences in cohort size. Orange, blue and red lines are used to delineate 2, 1 and 0.5 fold EMCA burden relative to the NCC cohort. The graph inset represents the raw number of variants at each gene between the OHRM and NCC cohort. (B) The number of rare pLoF variants from each cohort. (PPTX $73879 \mathrm{~kb}$ )

Additional file 5: Table S1. DiscovEHR and TCGA participant demographic information. Table S2. DiscovEHR and TCGA EMCA participant demographic information. Table S3. Distribution of stages between races and studies as a percentage*. Table S4. Distribution of grades across studies for EMCA*. Table S5. Total patient information for therapy and outcomes among DiscovEHR EMCA cohort. (DOCX 18 kb)

Additional file 6: Difference of proportions test. After binning variants into genes, a difference of proportions test was performed across all genes between variants in the EMCA and the control or OHRM cohort. $P$-values were adjusted for multiple tests using FDR. (XLSX $16 \mathrm{~kb}$ )

\section{Abbreviations}

EMCA: Endometrial cancer; NCC: Elderly non-cancer control cohort: OHRM: Other hormone related malignancy; TCGA: The cancer genome atlas; WES: Whole exome sequencing

\section{Acknowledgements}

We thank Raghu P. Metpally and Sarathbabu Krishnamurthy for preliminary analysis. In addition, we gratefully acknowledge the TCGA Consortium and all its members for the TCGA Project initiative, for providing sample, tissues, data processing and making data and results available. The results published here are in whole or part based upon data generated by The Cancer Genome Atlas pilot project established by the NCl and NHGRI. Information about TCGA and the investigators and institutions that constitute the TCGA research network can be found at http://cancergenome.nih.gov. We thank members of Regeneron for their comments and feedback. Finally, we thank the patient-participants of the MyCode community for being a part of the DiscovEHR research study.

\section{Funding}

This project is funded, in part, under a grant with the Pennsylvania Department of Health (\#SAP 4100070267). The Department specifically disclaims responsibility for any analyses, interpretations or conclusions. We gratefully acknowledge the funding support from Geisinger Medical Center (SRC-075) (RG) and Rice Women's Cancer Research Fund (RG and VRD). Support for this work also came from NHGRI T32HG009495-01 (JEM). The funders specifically disclaim responsibility for the study design, data collection, analyses, interpretation, conclusions, and writing of the manuscript.

\section{Availability of data and materials}

Additional information for reproducing the results described in the article is available from authors upon request. Availability of natural biological data from DiscovEHR cohort may subject to data user agreement.

\section{Authors' contributions}

JEM, VRD, DK, RG, and RPM conceived the project. Methodology was designed by JEM, TNP, RPM and SBK. Software was developed by JEM and TNP. DRL, AMC, and JEM curated data. JEM and MS performed the formal analysis along with visualization. DK provided resources. MDR, DK, RG, and RPM supervised all aspects of this work. The original draft was written by JEM and RG. JEM, RPM, TNP, SK, VRD, MS, DRL, AMC, DJC, MDR, DK, and RG reviewed and edited the manuscript. All authors have read and approved the manuscript

\section{Ethics approval and consent to participate}

The DiscovEHR cohort enrolled individuals from MyCode, which uses written consent and has previously been described [30]. This study was deemed to be exempt, therefore no further consent was needed. Geisinger Medical Ethics Committee - Geisinger Health System IRB protocol. "Phenotype validation and Biomarker Discovery Protocol for gynecological disorders". IRB number 2016-0119 was approved on 03/10/2016.

\section{Consent for publication}

Not applicable.

\section{Competing interests}

The authors declare that they have no competing interests.

\section{Publisher's Note}

Springer Nature remains neutral with regard to jurisdictional claims in published maps and institutional affiliations.

\section{Author details}

${ }^{1}$ Department of Genetics, Institute for Biomedical Informatics, Perelman School of Medicine, University of Pennsylvania, Philadelphia, PA 19104, USA ${ }^{2}$ Biomedical \& Translational Informatics Institute, Geisinger Health System, Danville, PA 17822, USA. ${ }^{3}$ Weis Center for Research, Geisinger Medical Center, Danville, PA 17822, USA. ${ }^{4}$ Huck Institute of the Life Sciences, Pennsylvania State University, University Park, PA 16802, USA. ${ }^{5}$ Department of Biostatistics, Epidemiology and Informatics, Perelman School of Medicine, University of Pennsylvania, Philadelphia, USA. ${ }^{6}$ Institute for Biomedical Informatics, University of Pennsylvania, Philadelphia, USA

Received: 30 August 2018 Accepted: 15 April 2019

Published online: 03 May 2019

\section{References}

1. Siegel RL, Miller KD, Jemal A. Cancer statistics, 2018. CA Cancer J Clin. 2018; 68:7-30.

2. Morice P, Leary A, Creutzberg C, Abu-Rustum N, Darai E. Endometrial cancer. Lancet. 2016;387:1094-108.

3. Braun MM, Overbeek-Wager EA, Grumbo RJ. Diagnosis and Management of Endometrial Cancer. Am Fam Physician. 2016;93:468-74.

4. Hoskins WJ. Principles and practice of gynecologic oncology: Lippincott Williams \& Wilkins; 2005. https://books.google.com/books?hl=en\&lr=\&id= 
KW9esgo759EC\&oi=fnd\&pg=PR11\&dq=Hoskins+WJ.+Principles+and +Practice+of+Gynecologic+Oncology.+Lippincott+Williams+\%26 +Wilkins\%3B+2005.\&ots=SLSte8vxah\&sig=Bo yVLKbFioPSTQMCZScbSa9Z3k\#v=onepage\&q=Hoskins\%20WJ. \%20Principles\%20and\%20Practice\%20of\%20Gynecologic\%20Oncology \%20Lippincott\%20Williams\%20\%26\%20Wilkins\%3B\%202005.\&f=false.

5. Fader AN, Arriba LN, Frasure HE, von Gruenigen VE. Endometrial cancer and obesity: epidemiology, biomarkers, prevention and survivorship. Gynecol Oncol. 2009;114:121-7.

6. Waldmann A, Eisemann N, Katalinic A. Epidemiology of malignant cervical, Corpus uteri and ovarian Tumours - current data and epidemiological trends. Geburtshilfe Frauenheilkd Georg Thieme Verlag KG. 2013;73:123-9.

7. Carlson MJ, Thiel KW, Leslie KK. Past, present, and future of hormonal therapy in recurrent endometrial cancer. Int J Women's Health. 2014;6: 429-35.

8. Okuda T, Sekizawa A, Purwosunu Y, Nagatsuka M, Morioka M, Hayashi M, et al. Genetics of endometrial cancers. Obstet Gynecol Int. 2010;2010:1-8,

9. Dunlop M. Cancer risk associated with germline DNA mismatch repair gene mutations. Hum Mol Genet. 1997;6:105-10.

10. Shai A, Segev Y, Narod SA. Genetics of endometrial cancer. Fam Cancer. 2014;13:499-505.

11. Strafford JC. Genetic testing for lynch syndrome, an inherited cancer of the bowel, endometrium, and ovary. Rev Obstet Gynecol. 2012;5:42-9.

12. Jones S, Stransky N, McCord CL, Cerami E, Lagowski J, Kelly D, et al. Genomic analyses of gynaecologic carcinosarcomas reveal frequent mutations in chromatin remodelling genes. Nature Communications. 2014:5:5006.

13. Alioto TS, Buchhalter I, Derdak S, Hutter B, Eldridge MD, Hovig E, et al. A comprehensive assessment of somatic mutation detection in cancer using whole-genome sequencing. Nature Communications. 2015;6:1-13.

14. Tamborero D, Gonzalez-Perez A, Perez-Llamas C, Deu-Pons J, Kandoth C, Reimand J, et al. Comprehensive identification of mutational cancer driver genes across 12 tumor types. Sci Rep. 2013;3:1-10.

15. Meyerson M, Gabriel S, Getz G. Advances in understanding cancer genomes through second-generation sequencing. Nat Rev Genet. 2010;11:685-96.

16. Knudson AG. Mutation and Cancer: statistical study of retinoblastoma. Proc Natl Acad Sci USA. 1971;68:820-3.

17. Saito M, Okamoto A, Kohno T, Takakura S, Shinozaki H, Isonishi S, et al. Allelic imbalance and mutations of the PTEN gene in ovarian cancer. Int. J. Cancer. 2000:85:160-5.

18. Nomoto S, Haruki N, Tatematsu Y, Konishi H, Mitsudomi T, Takahashi T. Frequent allelic imbalance suggests involvement of a tumor suppressor gene at $1 p 36$ in the pathogenesis of human lung cancers. Genes Chromosom Cancer. 2000:28:342-6.

19. Zhou C-Z, Qiu G-Q, Zhang F, He L, Peng Z-H. Loss of heterozygosity on chromosome 1 in sporadic colorectal carcinoma. World J Gastroenterol. 2004;10:1431-5.

20. Tomlinson IPM. Two hits revisited again. J Med Genet. 2001;38:81-5.

21. Miller EM, Patterson NE, Zechmeister JM, Bejerano-Sagie M, Delio M, Patel K, et al. Development and validation of a targeted next generation DNA sequencing panel outperforming whole exome sequencing for the identification of clinically relevant genetic variants. Oncotarget. 2017;8:102033-45.

22. Liang H, Cheung LWT, Li J, Ju Z, Yu S, Stemke-Hale K, et al. Whole-exome sequencing combined with functional genomics reveals novel candidate driver cancer genes in endometrial cancer. Genome Res. 2012;22:2120-9.

23. Lu C, Xie M, Wendl MC, Wang J, McLellan MD, Leiserson MDM, et al. Patterns and functional implications of rare germline variants across 12 cancer types. Nat Commun. 2015;6:1-13.

24. Kanchi KL, Johnson KJ, Lu C, McLellan MD, Leiserson MDM, Wendl MC, et al. Integrated analysis of germline and somatic variants in ovarian cancer. Nat Commun. 2014:5:1-29.

25. Stark Z, Dolman L, Manolio TA, Ozenberger B, Hill SL, Caulfied MJ, et al. Integrating Genomics into Healthcare: A Global Responsibility. Am J Human Genet. 2019;104:13-20.

26. Bastarache L, Hughey JJ, Hebbring S, Marlo J, Zhao W, Ho WT, et al. Phenotype risk scores identify patients with unrecognized Mendelian disease patterns. Science. 2018;359:1233-9.

27. Verma A, Lucas A, Verma SS, Zhang Y, Josyula N, Khan A, et al. PheWAS and beyond: the landscape of associations with medical diagnoses and clinical measures across 38,662 individuals from Geisinger. Am J Hum Genet. 2018; 102:592-608.
28. Verma A, Bang L, Miller JE, Zhang Y, Lee MTM, Zhang Y, et al. HumanDisease Phenotype Map Derived from PheWAS across 38,682 Individuals. Am J Hum Genet. 2019;104:55-64.

29. Long B, Lilyquist J, Weaver A, Hu C, Gnanaolivu R, Lee KY, et al. Cancer susceptibility gene mutations in type I and II endometrial cancer. Gynecol Oncol. 2019;152:20-5.

30. Carey DJ, Fetterolf SN, Davis FD, Faucett WA, Kirchner HL, Mirshahi U, et al. The Geisinger MyCode community health initiative: an electronic health record-linked biobank for precision medicine research. Genet Med. 2016;18: 906-13.

31. Dewey FE, Murray MF, Overton JD, Habegger L, Leader JB, Fetterolf SN, et al. Distribution and clinical impact of functional variants in 50,726 wholeexome sequences from the DiscovEHR study. Science. 2016;354. https://doi. org/10.1126/science.aaf6814.

32. McKenna A, Hanna M, Banks E, Sivachenko A, Cibulskis K, Kernytsky A, et al. The genome analysis toolkit: a MapReduce framework for analyzing nextgeneration DNA sequencing data. Genome Res Cold Spring Harbor Lab. 2010;20:1297-303.

33. Carson AR, Smith EN, Matsui H, Brækkan SK, Jepsen K, Hansen J-B, et al. Effective filtering strategies to improve data quality from population-based whole exome sequencing studies. BMC Bioinformatics. 2014;15:125.

34. Van Allen EM, Wagle N, Stojanov P, Perrin DL, Cibulskis K, Marlow S, et al. Whole-exome sequencing and clinical interpretation of formalin-fixed, paraffin-embedded tumor samples to guide precision cancer medicine. Nat Med. 2014:20:682-8.

35. Landrum MJ, Lee JM, Riley GR, Jang W, Rubinstein WS, Church DM, et al. ClinVar: public archive of relationships among sequence variation and human phenotype. Nucleic Acids Res. 2014:42:D980-5.

36. McLaren W, Pritchard B, Rios D, Chen Y, Flicek P, Cunningham F. Deriving the consequences of genomic variants with the Ensembl API and SNP effect predictor. Bioinformatics. 2010;26:2069-70

37. McLaren W, Gil L, Hunt SE, Riat HS, Ritchie GRS, Thormann A, et al. The Ensembl variant effect predictor. Genome Biol. 2016;17:122.

38. Lek M, Karczewski KJ, Minikel EV, Samocha KE, Banks E, Fennell T, et al. Analysis of protein-coding genetic variation in 60,706 humans. Nature. 2016; 536:285-91.

39. Fu W, O'Connor TD, Jun G, Kang HM, Abecasis G, Leal SM, et al. Analysis of 6,515 exomes reveals the recent origin of most human protein-coding variants. Nature. 2013;493:216-20.

40. Consortium GP. A global reference for human genetic variation. Nature. 2015;526:68-74

41. Richards S, Aziz N, Bale S, Bick D, Das S, Gastier-Foster J, et al. Standards and guidelines for the interpretation of sequence variants: a joint consensus recommendation of the American College of Medical Genetics and Genomics and the Association for Molecular Pathology. Genet Med. 2015;17:405-23.

42. Schmandt RE, Iglesias DA, Co NN, Lu KH. Understanding obesity and endometrial cancer risk: opportunities for prevention. Am J Obstet Gynecol. 2011;205:518-25.

43. Basen-Engquist K, Chang M. Obesity and Cancer risk: recent review and evidence. Curr Oncol Rep. 2010;13:71-6.

44. Hamilton CA, Cheung MK, Osann K, Chen L, Teng NN, Longacre TA, et al. Uterine papillary serous and clear cell carcinomas predict for poorer survival compared to grade 3 endometrioid corpus cancers. Br J Cancer. 2006;94:642-6.

45. Forbes SA, Bindal N, Bamford S, Cole C, Kok CY, Beare D, et al. COSMIC: mining complete cancer genomes in the catalogue of somatic mutations in Cancer. Nucleic Acids Res Narnia. 2011;39:D945-50.

46. Randall LM, Pothuri B. The genetic prediction of risk for gynecologic cancers. Gynecol Oncol. 2016;141:10-6.

47. Levine DA, Network TCGAR. Integrated genomic characterization of endometrial carcinoma. Nature. 2013;497:67-73.

48. Lee $\mathrm{S}$, Abecasis GR, Boehnke M, Lin X. Rare-Variant Association Analysis: Study Designs and Statistical Tests. Am J Human Genet. 2014;95:5-23.

49. Bell D, O'Hara. The genomics and genetics of endometrial cancer. AGG. 2012;2012(2):33-15.

50. Park S, Supek F, Lehner B. Systematic discovery of germline cancer predisposition genes through the identification of somatic second hits. Nat Commun. 2018:9:302-13.

51. Musgrove EA, Caldon CE, Barraclough J, Stone A, Sutherland RL. Cyclin D as a therapeutic target in cancer. Nature. 2011;11:558-72.

52. Ikeda Y, Oda K, Ishihara H, Wada-Hiraike O, Miyasaka A, Kashiyama T, et al. Prognostic importance of CDK4/6-specific activity as a predictive marker for 
recurrence in patients with endometrial cancer, with or without adjuvant chemotherapy. Br J Cancer. 2015;113:1477-83.

53. Yen C-F, Liao S-K, Huang SJ, Tabak S, Arcuri F, Lee C-L, et al. Decreased endometrial expression of leukemia inhibitory factor receptor disrupts the STAT3 signaling in Adenomyosis during the implantation window. Reprod Sci. 2017;24:1176-86.

54. Cheng J-G, Chen JR, Hernandez L, Alvord WG, Stewart CL. Dual control of LIF expression and LIF receptor function regulate Stat3 activation at the onset of uterine receptivity and embryo implantation. Proc Natl Acad Sc USA. 2001;98:8680-5.

55. Bennett JA, Oliva E, Nardi V, Lindeman N, Ferry JA, Louissaint A. Primary endometrial marginal zone lymphoma (MALT lymphoma). Am J Surg Pathol. 2016:40:1217-23.

56. Kastrinos F, Stoffel EM. History, genetics, and strategies for Cancer prevention in Lynch syndrome. Clin Gastroenterol Hepatol. 2014:12:715-27.

57. Periyasamy M, Singh AK, Gemma C, Kranjec C, Farzan R, Leach DA, et al. p53 controls expression of the DNA deaminase APOBEC3B to limit its potential mutagenic activity in cancer cells. Nucleic Acids Res. 2017;45:11056-69.

58. Chiu Y-L, Greene WC. The APOBEC3 Cytidine Deaminases: An Innate Defensive Network Opposing Exogenous Retroviruses and Endogenous Retroelements. Ann Rev. 2008;26:317-53. https://doi.org/10.1146/annurev. immunol.26.021607.090350.

59. Refsland EW, Harris RS. The APOBEC3 Family of Retroelement Restriction Factors. Intrinsic Immunity. Berlin: Springer, Berlin, Heidelberg; 2013. p. 1-27.

60. Srsen V, Gnadt N, Dammermann A, Merdes A. Inhibition of centrosome protein assembly leads to p53-dependent exit from the cell cycle. J Cell Biol. 2006;174:625-30.

61. Balczon R. PCM-1, a 228-kD centrosome autoantigen with a distinct cell cycle distribution. J Cell Biol. 1994;124:783-93.

62. Reiter A, Walz C, Watmore A, Schoch C, Blau I, Schlegelberger B, et al. The t $(8 ; 9)($ p22;p24) is a recurrent abnormality in chronic and acute leukemia that fuses PCM1 to JAK2. Cancer Res. 2005;65:2662-7.

63. Adelaide J, Perot C, Gelsi-Boyer V, Pautas C, Murati A, Copie-Bergman C, et al. A t $(8 ; 9)$ translocation with PCM1-JAK2 fusion in a patient with T-cell lymphoma. Leukemia. 2006;20:536-7.

64. Segev Y, lqbal J, Lubiński J, Gronwald J, Lynch HT, Moller P, et al. The incidence of endometrial cancer in women with BRCA1 and BRCA2 mutations: an international prospective cohort study. Gynecol Oncol. 2013;130:127-31.

65. Seroz T, Hwang JR, Moncollin V, Egly JM. TFIIH: a link between transcription, DNA repair and cell cycle regulation. Curr Opin Genet Dev. 1995;5:217-21.

66. von Bergh ARM, Beverloo HB, Rombout $P$, van Wering $E R$, van Weel $M H$, Beverstock GC, et al. LAF4, an AF4-related gene, is fused to MLL in infant acute lymphoblastic leukemia. Genes Chromosomes Cancer. 2002;35:92-6.

67. Moore JM, Oliver PL, Finelli MJ, Lee S, Lickiss T, Molnár Z, et al. Laf4/Aff3, a Gene Involved in Intellectual Disability, Is Required for Cellular Migration in the Mouse Cerebral Cortex. PLoS ONE. 2014:9:e105933.

68. Dong X-Y, Guo P, Sun X, Li Q, Dong J-T. Estrogen up-regulates ATBF1 transcription but causes its protein degradation in estrogen receptor-alphapositive breast cancer cells. J Biol Chem. 2011;286:13879-90.

69. Minamiya Y, Saito H, Ito M, Imai K, Konno H, Takahashi N, et al. Suppression of zinc finger Homeobox 3 expression in tumor cells decreases the survival rate among non-small cell lung cancer patients. Cancer Biomark. 2012;11:139-46.

70. Huang S, Ernberg I, Kauffman S. Cancer attractors: a systems view of tumors from a gene network dynamics and developmental perspective. Semin Cell Dev Biol. 2009;20:869-76.

Ready to submit your research? Choose BMC and benefit from:

- fast, convenient online submission

- thorough peer review by experienced researchers in your field

- rapid publication on acceptance

- support for research data, including large and complex data types

- gold Open Access which fosters wider collaboration and increased citations

- maximum visibility for your research: over $100 \mathrm{M}$ website views per year

At BMC, research is always in progress.

Learn more biomedcentral.com/submissions 